\title{
UNA SOCIETÀ SENZA CAVALLERIA? \\ IL GIUDICATO DI ARBOREA E LA CORONA DI ARAGONA TRA XIV E XV SECOLO
}

\author{
LUCIANO GALLINARI ${ }^{1}$
}

\begin{abstract}
Riassunto: La finalità di questo articolo è di verificare l'esistenza di istituzioni militari cavalleresche e i loro possibili riflessi sulla società del Giudicato di Arborea nei secoli $\mathrm{XIV}-\mathrm{XV}$, quando esso fu impegnato in una lunga guerra contro il Re d'Aragona, signore feudale del Regnum Sardiniae et Corsicae.

Parole chiave: Aragona; Arborea; Cavalleria.
\end{abstract}

Abstract: The aim of this article is to verify the existence of military institutions as the Chivalry and its possible aspects in the society of the Arboreas Iudicatus in the XIVthXVth Century, when it was engaged in a long war against the King of Aragon, holder of the feodal lordship of the Regnum Sardiniae et Corsicae.

Keywords: Aragon, Arborea, Chivalry.

Già in altri nostri lavori ci siamo occupati della struttura dell'esercito giudicale arborense tra la fine del Trecento e i primi anni del Quattrocento e dei suoi riflessi nella società indigena sarda ${ }^{2}$. Più volte ci siamo domandati se fosse possibile rinvenire nel Giudicato d'Arborea una classe di milites omologhi a quelli che agivano in pressoché tutti i campi di battaglia dell'Europa occidentale degli ultimi secoli del Medioevo.

Il nostro interesse va aldilà del semplice aspetto tecnico-militare e si concentra soprattutto sui tentativi di analisi di eventuali peculiarità riscontrabili nella società giudicale. In particolar modo, nel presente lavoro intendiamo

\footnotetext{
${ }^{1}$ Istituto di Storia dell'Europa Mediterranea, del Consiglio Nazionale delle Ricerche, Sezione Rapporti italo-iberici, Cagliari, Italia. 2003.

Data di recezione dell'articolo: giugno 2003. Data di accettazione e versione finale: luglio

${ }^{2}$ L. GALlinARI, Guglielmo III di Narbona, ultimo sovrano d'Arborea, e la guerra dei Cent'anni, in "Medioevo. Saggi e rassegne", 18 (1993), pp. 91-121; ID., Riflessi della guerra tra Arborea e Aragona alla corte del re di Francia: nuove acquisizioni documentarie e prospettive di ricerca, in "Medioevo. Saggi e rassegne", 22 (1997), pp. 149-172; ID., Preliminary research on the Intervention of France in the War between the Kingdom of Arborea and the Crown of Aragon around 1400, in "Nottingham Medieval Studies", XLIII (1999), pp. 152-171, e ID., Gli ultimi anni di esistenza del Giudicato di Arborea: Riflessioni e prospettive di ricerca, in "Medioevo. Saggi e rassegne", Cagliari, 25 (2001), in corso di stampa.

«Anuario de Estudios Medievales», 33/2 (2003), pp. 849-879 .- ISSN 0066-5061.
} 
soffermarci su due temi e su due periodi storici. In primo luogo, vogliamo analizzare alcune testimonianze letterarie e cronachistiche che collegarono il Giudicato di Arborea alla cultura cortese e cavalleresca nel corso del XII-XIII secolo. In seconda battuta, invece, concentreremo la nostra attenzione sulla seconda metà del XIV secolo, durante la quale la conduzione dell'attività politica del Giudicato di Arborea fu assunta da Mariano IV e, de facto, da Brancaleone Doria. La motivazione di questa nostra scelta risiede nel fatto che crediamo che, anche nei documenti di questo secondo periodo storico, si possano riscontrare diversi dati che consentono di formulare alcune ipotesi interpretative sul tema dell'assetto sociale giudicale. E ciò in relazione all'attività bellica, ridivenuta di nuovo intensa dopo la liberazione del conte di Monteleone dalla prigionia catalano-aragonese di Castel di Cagliari nel gennaio 1390.

Nel dettaglio, alcuni di questi dati a cui alludiamo e che analizzeremo in seguito ci sembra che facciano intravvedere come certi elementi appartenenti alla cultura cavalleresca nel senso più tradizionale del termine dovettero penetrare e sopravvivere nella società giudicale arborense. E questo è un dato su cui vale la pena di soffermarsi per un duplice ordine di motivi. In prima istanza, perché il Giudicato d'Arborea continuava a mostrare una struttura sociale per molti versi peculiare, che risentiva senz'ombra di dubbio della sua originale storia precedente e, in secondo luogo, perché nel periodo al centro della nostra attenzione - la seconda metà del XIV secolo - era impegnato in una contrapposizione militare a oltranza con un avversario molto più forte da un punto di vista economico e militare quale la Corona d'Aragona ${ }^{3}$.

${ }^{3}$ Il Giudicato di Arborea -l'ultimo dei quattro stati indigeni che si crearono dalla frammentazione dell'antica provincia bizantina di Sardegna, forse tra X e XI secolo- deve la peculiarità della propria struttura sociale alle sue radici che affondano nel mondo greco-bizantino dell'Alto Medioevo. In effetti, la società arborense ancora alla fine del XIV secolo -il periodo da noi esaminato adesso- continuava ad avere tracce consistenti della sua matrice orientale che qui sarebbe troppo lungo e fuorviante accennare. In questa sede ci occuperemo solo di quegli elementi che appaiono in relazione con il tema da noi trattato. Sulle modalità e la cronologia della creazione dei Giudicati rimandiamo a E. BESTA, La Sardegna medioevale. 2. Le istituzioni politiche, economiche, giuridiche, sociali, Bologna, 1979, pp. 9-11 per il quale la quadripartizione della Sardegna sarebbe avvenuta forse oltre il 1000, e sarebbe stata causata da rivolgimenti interni scaturiti da forze endogene. Di parere diverso invece A. SolmI, Studi storici sulle istituzioni della Sardegna nel Medioevo, (M.E. Cadeddu, ed.), Nuoro, 2001, p. 57 secondo il quale il vincolo di dipendenza con l'Oriente dovette interrompersi molto prima dell 'XI secolo, per cui l'origine dei Giudicati doveva essere fatta risalire all'VIII o al IX secolo. Favorevole a quest'ultima ipotesi si mostra anche F.C. CASUlA, La Sardegna aragonese. 1 La Corona d'Aragona, Sassari, 1990, pp. 89-90 e 125. S. PETRUCCI, "Storia politica e istituzionale della Sardegna medioevale (secoli XI-XIV)", in Il Medioevo. Dai Giudicati agli Aragonesi, Storia dei Sardi e della Sardegna, vol. II, Milano, 1987, pp. 99-101 si mostra incline a individuare il momento culminante del processo istituzionale che portò alla nascita degli iudices ai secoli X-XI, 
Iniziamo adesso a esaminare quali sono i termini che ci hanno indotti a effettuare la nostra analisi e le fonti che abbiamo preso in considerazione. In linea di massima, possiamo dire che per le testimonianze più antiche si tratta di due delle rarissime fonti letterarie del Medioevo sardo; mentre al contrario, per il secondo periodo, si tratta di documenti prodotti da ufficiali aragonesi operanti in Sardegna che furono inseriti nel processo di ribellione e lesa maestà intrapreso dai re iberici nei confronti dei giudici arborensi a partire dalla metà del XIV secolo ${ }^{4}$. Qualche altra informazione la ricaviamo anche da fonti sarde in originale o inserite in copia negli atti del suddetto processo e altrimenti andate perdute.

Quanto detto deve farci subito sottolineare che, data la provenienza di parte delle fonti in nostro possesso, è quanto mai necessario procedere con estrema cautela nel fare affermazioni, anche perché il tema da noi trattato di per sé ha favorito il nascere di diverse interpretazioni.

Innanzitutto, per comprendere meglio i dati che esamineremo, riteniamo opportuno fare un breve quadro degli avvenimenti che caratterizzarono il XIV secolo per il Giudicato di Arborea. Dal 1323 il sovrano d'Aragona Giacomo II il Giusto aveva iniziato la realizzazione concreta di quello che fino a quel momento era stato solo sulla carta il Regnum Sardiniae et Corsicae, creato il 4 aprile 1297 dal papa Bonifacio VIII e infeudato al monarca iberico come ricompensa per aver rinunciato alla Sicilia ${ }^{5}$. Dopo circa

dal momento che fino ad allora il massimo governante sardo era indicato con il titolo bizantino di $\dot{\alpha} \rho \chi \omega \nu$. Nel presente lavoro l'autore insisteva molto sull'importanza del fenomeno di regionalizzazione del potere, dovuto alla rarefazione dei rapporti con Bisanzio, come risulterebe confermato anche da esiti istituzionali simili a quelli sardi, verificatisi anche nelle altre compagini italiche appartenute all'impero, e come risulterebbe anche comprovato dall'affermarsi di una sola famiglia - L Lacon- alla base di tutte e quattro le dinastie giudicali dell'XI secolo.

${ }^{4} \mathrm{Nel}$ dettaglio, le fonti consultate sono il Libellus Judicum turritanorum (A. Sanna, ed.), Cagliari, 1957, una cronaca redatta in sardo logudorese nella seconda metà del XIII secolo, che descrive gli eventi del Giudicato di Torres o Logudoro dal 1065 fino alla scomparsa dello Stato indigeno nel 1259; e la Memoria de las cosas que han aconteçido en algunas partes de Çerdeña (P. Maninchedda, ed.), Cagliari, 2000, una cronaca elaborata con ogni probabilità nel convento di S. Francesco dei Minori di Cagliari che viene attribuita al XVI secolo, ma che in realtà a detta del suo editore è una sorta di silloge di testi medievali. Per quanto riguarda invece le fonti documentarie analizzate, i Procesos contra los Arborea sono una serie composta da dieci registri contenenti le copie degli atti del processo feudale per ribellione e lesa maestà avviato dai funzionari regi in origine contro il giudice di Arborea Mariano IV nel 1353. I procedimenti legali, che riflettono l'evolversi dei rapporti politici tra il Regno di Sardegna e Corsica e il Giudicato di Arborea, furono proseguiti anche nei confronti dei successori del giudice oristanese giungendo fino agli anni 1392-93.

${ }^{5} \mathrm{Su}$ Giacomo II il Giusto e i numerosi problemi connessi alla realizzazione pratica della conquista della Sardegna rimandiamo al recente saggio di M.E. CADEDDU, Giacomo II d'Aragona e la conquista del regno di Sardegna e Corsica, in "Medioevo. Saggi e rassegne", 20 (1995), pp. 251-316, e all'abbondante e aggiornata bibliografia in esso contenuta, in cui l'autrice riordina in 
trent'anni di rapporti tutto sommato buoni tra il nuovo Regnum e il Giudicato di Arborea - la principale entità statuale isolana- dal 1353 il sovrano giudicale Mariano IV aveva iniziato un'attività di contrapposizione bellica nei confronti dei Catalano-Aragonesi. Le motivazioni che spinsero il giudice a fare ciò sono espresse chiaramente nei documenti inseriti nei Procesos a cui alludevamo prima ${ }^{6}$. Di fatto, a partire dalla metà del secolo il Giudicato si trovò a dover fronteggiare con le armi una compagine decisamente più potente sia dal punto di vista economico sia da quello politico-militare. In considerazione di quanto detto, si può ammettere senza difficoltà che questo confronto armato poté produrre o accelerare cambiamenti nell'organizzazione bellica giudicale.

Come sempre, anche a proposito di questo tema della storia medioevale sarda, occorre fare i conti con una notevole penuria di fonti. I dati i nostro possesso, tuttavia, ci consentono di formarci un'idea approssimativa di come doveva essere composto l'esercito giudicale e di che tipo di immagine esso dovesse dare all'esterno.

In generale, dalla lettura delle fonti catalano-aragonesi sembra che sia possibile ricavare una duplice serie di elementi: da una parte, la constatazione di un cospicuo numero di armati alle dipendenze del giudice arborense;

modo sistematico la complessa questione che costituisce da almeno un secolo uno dei principali leit-motiv della storiografia catalana e sarda.

${ }^{6}$ Risulta estremamente interessante sotto quest'ottica confrontare le due versioni dell'inizio delle ostilità tra Sardi e Iberici. Mariano IV in una sua lettera del 18 dicembre 1353 a sua sorella, Bonaventura d'Arborea, la informò dei problemi insorti con Bernat de Cabrera, luogotenente regio in Sardegna, il quale lo aveva dipinto come suspectus et livorosus agli occhi del re Pietro IV, al fine di potergli portare via tutti i beni e distruggere così la sua Casata. Decisamente diversa invece la testimonianza di Saladino, vescovo di Dolia, il quale tra le altre cose spiegò che Mariano IV aveva iniziato le ostilità contro i Catalani quia domini cathalani volebant eum privare regno suo quod tenet propter quod idem iudex impugnat taliter cathalanos ut melius se possit defendere ab eodem. Cfr. Archivo de la Corona de Aragón, Cancillería (d'ora in poi ACA, $C$ ), Procesos contra los Arborea, vol. III, ff. $101 \mathrm{r}$-v per la lettera del giudice d'Arborea e vol. V, ff. $75 \mathrm{v}-76 \mathrm{v}$ per la deposizione del vescovo di Dolianova.

${ }^{7}$ Un esempio di truppe professionali arruolate dai giudici di Arborea ci è fornito dalla testimonianza del governatore aragonese di Sardegna in merito allo scontro aperto tra Mariano IV e suo fratello Giovanni d'Arborea, originatosi per le posizioni filocatalane del secondo. In questo caso, l'ufficiale iberico affermò che tutti e due tra i propri schieramenti potevano annoverare gentes extranee nationis, Tehotonici videlicet et Toschani. Situazione simile anche alcuni anni dopo, alla ripresa delle ostilità aperte dei Giudicali contro gli Aragonesi. Il patrono genovese Luiso Riccio rilasciò una testimonianza il 7 novembre 1365 secondo la quale dicitur et est fama quod dictus iudex Arboree fecit venire in dictam insulam per partes civitatis pissane equites et pedites englenses et aliarum extranearum gencium, et quod predicta fuerunt facta patenter et notorie prout ipse testis vidit [...]. Sulla falsariga, anche la testimonianza di Bernardo di Santo Noxero rilasciata il 30 dic.1366, il quale vidit in campo iudicis Arboree in villa Sent Luri et in villa Salargii gentes armigeras extranee nacionis videlicet inglenses, teotonicos, lombardos et tuschos. Cfr. ACA, C, Procesos contra los Arborea, voll. II, f. 31v, e VIII, ff. 18r-v e 56v. 
dall'altra, la parallela affermazione che nonostante ciò il sovrano indigeno non dovesse essere considerato un nemico temibile. E a questo proposito, pur considerando un'inevitabile parzialità da parte iberica, vi è da dire che in occasione dei principali scontri aperti tra Sardi e Iberici, i primi ebbero praticamente sempre la peggio, pur essendo spesso in grande vantaggio numerico ${ }^{8}$. E questo dovrebbe essere un campanello di allarme per lo storico, al quale dovrebbe suggerire di soffermarsi con attenzione sull'apparato militare giudicale.

Vediamo adesso alcune di queste testimonianze documentarie e che tipo di informazioni ci offrono. Iniziamo dall'immagine che potevano avere i Catalano-Aragonesi dei loro contendenti isolani, tenendo in conto che a opporsi alla loro azione politica non vi furono solo i giudici arborensi ma anche gli esponenti della grande famiglia di origine ligure dei Doria, detentori di enormi possedimenti territoriali nella parte nord-occidentale della Sardegna. Se osserviamo diacronicamente i dati offerti dalle nostre fonti, in essi vediamo chiaramente un riflesso degli eventi.

Per esempio, i commissari incaricati di istituire il processo di ribellione contro Mariano IV, in riferimento al suo comportamento in occasione della battaglia di Aidu de Turdu (1347), nella quale egli intervenne per salvare quanto rimaneva delle truppe iberiche decimate da un agguato condotto dalle milizie al soldo dei Doria, così si espressero: est notorium et manifestum in insula supradicta et etiam extra eam omnibus noticiam habentibus de eadem dictum iudicem fore longe potentiorem gentibus et divitis a longo tempore citra et sui predecessores quam rebelles de Auria supradicti [il grassetto è nostro] ${ }^{9}$.

In realtà, però, a conferma delle difficoltà di una corretta interpretazione di queste fonti documentarie, non tutti si dichiaravano d'accordo con questa valutazione fatta dai commissari regi. Dalmau de Rodegia, mercante di Castel di Cagliari, per esempio, riferì di aver sentito dire che barones de

${ }^{8} \mathrm{Un}$ esempio potrebbe essere dato dall'assedio posto dalle truppe giudicali arborensi a Castel di Cagliari nell'estate 1353. Finché si trattò di compiere incursioni negli orti vicini alle mura della capitale aragonese e di avere qualche scaramuccia con gli Iberici, le truppe sarde - accampate nella villa di Selargius, a qualche chilometro di distanza- riuscirono ad avere le meglio. Quando però sopraggiunse con il grosso delle milizie iberiche l'ammiraglio Bernat de Cabrera in poco tempo riusci a mettere in fuga gli isolani, strappando loro anche gli stendardi, nonostante che secondo la testimonianza di Mariano Frau, la consistenza dell'esercito giudicale a Sanluri, fosse di equites circa sexcentos et pedites duodecim milia et ultra. Per questi eventi vedasi ACA, C, Procesos contra los Arborea, vol. VI, ff. 101r-103v e 178v-179v.

${ }^{9} \mathrm{ACA}, C$, Procesos contra los Arborea, vol. II, f. $32 \mathrm{v}$. 
Auria habebant meliores et audaciores homines quo ad armorum exercicium quam iudex predictus, mentre invece Bernat Salou, corsaro, abitante della capitale informò di un'altra voce comune, secondo la quale dictus iudex est potencior quam aliquis de insula Sardinie super in eadem insula giungendo a sostenere - con un pizzico di esagerazione, anche se si riferiva solo alle truppe iberiche di stanza nell'isola - che il giudice fosse più potente dello stesso sovrano aragonese. Di opinione simile anche Guillem Tarrades, abitante di Castel di Cagliari, il quale dixit se audivisse dici in Castro Callari cum aliis pluribus partibus insule Sardinie publice quod dictus iudex est potentior gentibus et diviciis rebellibus de Auria predictis [il grassetto è nostro $]^{10}$.

Se analizziamo attentamente queste testimonianze appena citate, possiamo estrapolarne alcuni dati interessanti. Pur nella diversità delle opinioni formulate, i testimoni sembrano fornire due tipi di giudizi: per quanto riguarda il giudice d'Arborea, essi mettono l'accento su'ia sua ricchezza che si traduce anche nel maggior numero di armati a sua disposizione rispetto ai Doria ${ }^{11}$. Quanto a questi ultimi, però, sembrava che essi potessero disporre di meliores et audaciores homines quo ad armorum exercicium. Giudizio interessante, questo, che dovremo tenere in considerazione quando andremo a commentare più nel dettaglio le caratteristiche dell'esercito giudicale. Giudizio che forse dovremmo ricollegare a quanto abbiamo già osservato in nostri precedenti lavori a proposito delle caratteristiche tecnico-tattiche dei soldati giudicali ${ }^{12}$.

Fermo restando il valore e l'impegno personale profuso dai militi sardi che pagarono con la vita i combattimenti con gli Iberici, ci sembra che fino all'ultimo grande scontro armato tra Arborensi e Aragonesi -la battaglia di Sanluri del giugno 1409- si possano individuare nella struttura dell'esercito indigeno sardo alcune peculiarità che lo resero differente da quello a esso contrapposto e che, forse, concorrono a spiegare i motivi della sconfitta dello stato giudicale.

${ }^{10} \mathrm{ACA}, C$, Procesos contra los Arborea, vol. II, ff. 37r, 72r e 74 v.

${ }^{11}$ Sulla ricchezza dei giudici di Arborea che ritorna come una sorta di leit-motiv nelle fonti tra XIII e XV secolo, vedasi anche quanto sostenuto a proposito delle disponibilità economiche di Ugone II e del suo grande potere di corruzione nei confronti dei Catalano-Aragonesi in Memoria de las cosas, p. L.

${ }^{12} \mathrm{Cfr}$. da ultimo L. Gallinari, Gli ultimi anni di esistenza, in corso di stampa, e la bibliografia lì contenuta.

«Anuario de Estudios Medievales», 33/2 (2003), pp. 849-879 .- ISSN 0066-5061. 
Torniamo adesso all'immagine delle truppe giudicali. Quanto detto sopra a proposito della scarsa considerazione di cui esse godevano presso gli Iberici può essere confermato dalle affermazioni di Rambau de Corbera, governatore del Regno di Sardegna. Egli, il $1^{\circ}$ novembre 1353, scrisse all'ammiraglio della flotta iberica, Bernat de Cabrera, per comunicargli che il giudice Mariano IV e Matteo Doria, capo della potente consorteria sardoligure, erano comparsi sotto le mura di Sassari e vi avevano posto un breve assedio. Ciò che ci interessa rimarcare è che in quel frangente il giudice era accompagnato da 400 uomini a cavallo e 1000 a piedi, i quali a detta dell'ufficiale catalano valen poch ${ }^{13}$. Anche in questo caso, riteniamo che, pur ammettendo un po' di spavalderia da parte del funzionario iberico, egli non avrebbe corso più rischi del necessario se avesse valutato un pericolo maggiore. Forse, questa sicurezza del governatore si basava su due ordini di fattori: il primo, costituito probabilmente dall'armamento che questi soldati giudicali avevano in dotazione; il secondo, invece, da questa fama di non buoni combattenti che accompagnava i militari isolani, almeno agli occhi degli iberici ${ }^{14}$. Di certo, questa spavalderia non apparirebbe spiegabile se ci si limitasse a considerare il numero non certo esiguo dei militari al seguito del giudice arborense in quell'occasione.

A questo punto occorre soffermarsi sulle notizie in nostro possesso che riguardano l'assetto dell'esercito giudicale.

Le fonti documentarie finora note menzionano ripetutamente la presenza di fanti e uomini a cavallo che operavano in tutti i teatri della guerra contro i Catalano-Aragonesi; a questi dati che non sembrano connotare in alcun modo particolare le truppe giudicali, occorre aggiungere la quasi

\footnotetext{
${ }^{13}$ ACA, C, Procesos contra los Arborea, vol. III, ff. 95v-96r.

${ }^{14}$ Troviamo interessante questo capovolgimento di immagine dei combattenti sardi agli occhi di spettatori esterni all'isola, se confrontiamo questa valutazione del governatore aragonese del XIV secolo e i cronisti arabi dell'Alto Medioevo che ripetutamente sottolinearono il carattere bellicoso e feroce dei Sardi, che avrebbe permesso loro di impedire lunghi stanziamenti musulmani nell'isola. Valga come esempio l'attacco islamico contro la Sardegna degli anni 821-2 nel corso del quale secondo il cronista arabo Ibn Al Athir «i Musulmani effettuarono dall'Ifriqiyah un'incursione contro l'isola della Sardegna dove massacrarono infedeli e da loro vennero massacrati...». Altrettanto interessante risulta la testimonianza del famoso geografo arabo $\mathrm{Al}$ Idrisi, che nella sua opera Nuzhat Al Mushtaq Fî Ikhtiraq Al-Afaq, scritta per il re normanno di Sicilia Ruggero II, riportava che i Sardi erano «gente di proposito e valorosa, che non lascia mai l'arme». Cfr. la traduzione in lingua italiana effettuata da M. AMARI, Storia dei Musulmani di Sicilia, vol. I, Catania, 1933, pp. 49-51. Non è del tutto d'accordo con questa traduzione M.M BAZAMA, Arabi e sardi nel medioevo, Cagliari, 1988, pp. 109-110 e 162 che propone invece: «[i Sardi] sono pronti al richiamo d'aiuto, combattenti, decisivi e mai si separano dalle loro armi (intende guerrieri nati)».
} 
onnipresenza della virga sardisca, una vera e propria arma nazionale sarda del Basso Medioevo ${ }^{15}$. Per completare il quadro degli elementi peculiari non si può trascurare di menzionare le "mute": dalle fonti documentarie sappiamo che l'esercito arborense era costituito da elementi provenienti da ogni villaggio giudicale che era stato suddiviso in tre parti, le quali si alternavano al fronte ${ }^{16}$.

Elencate queste peculiarità dell'esercito giudicale, riteniamo opportuno soffermarci adesso sulla presenza di combattenti a cavallo. In un nostro recente lavoro abbiamo già evidenziato la presenza nelle fonti documentarie del XIV secolo di esponenti dei lieros ab equo o lieros de cavallu, uomini liberi che avevano l'obbligo di servire lo stato con un cavallo di un determinato valore ${ }^{17}$.

Acluni studiosi ritengono che sia possibile ipotizzare un collegamento tra costoro e i $K \alpha \beta \alpha \lambda \lambda \dot{\alpha} \rho$ ıo bizantini, stratioti che militavano con un cavallo proprio e che ricevevano in cambio dall'Impero una sorta di "feudo" militare e che godevano di immunità fiscale. In base a tale ipotesi interpretativa, la presenza di questo ceto di soldati-agricoltori risulterebbe confermata dall'esistenza di moltissimi toponimi areali in lingua sarda tipo kaddaris/quaddaris, che sarebbero la traduzione del termine greco $\mathrm{K} \alpha \beta \dot{\alpha} \lambda \lambda \alpha \rho \mathrm{\imath} \varsigma^{18}$.

$\mathrm{Su}$ questa teoria sono state avanzate alcune riserve che riteniamo interessanti, anche ai fini di un discorso che stiamo conducendo intorno alla diffusione e alla durata dei legami che la Sardegna ebbe con la cultura bizantina. Legami che possono contribuire a comprendere meglio l'assetto che la società giudicale sarda ebbe ancora nel XIV e XV secolo quando dovette confrontarsi militarmente con la Corona d'Aragona.

\footnotetext{
${ }^{15}$ Sulla virga sardisca e le diverse ricostruzioni di quest'arma dai molteplici usi per i sardi giudicali, rimandiamo a G. FoIS, Un'arma medioevale sarda: la 'virga', in "Quaderni bolotanesi”, 21 (1995), p. 201 che riassume anche precedenti tentativi di ricostruzione.

${ }^{16} \mathrm{Su}$ questa peculiarità militare abbiamo la testimonianza di Pietro Cotza, abitante di Sinnai a pochi chilometri di Castel di Cagliari, il quale raccontò che il comandante dell'esercito giudicale faciebat mudas in exercitu suo de certis hominibus equitum et peditum de septimana in septimanam. La finalità ultima di questo sistema era di non abbandonare del tutto le altre attività che garantivano la sopravvivenza economica delle comunità del Giudicato. Cfr. ACA, $C$, Procesos contra los Arborea, vol. VI, ff. 116v-118v. Sul tema delle mudas rimandiamo a F.C. CASULA, La Sardegna aragonese, 1, pp. 279-281.

${ }^{17}$ ACA, C, Procesos contra los Arborea, vol. VI, f. 120r: Petrum Stavinum qui solebat esse de liberis ab equo pisani comunis [il grassetto è nostro].

${ }^{18} \mathrm{G}$. PAULIS, Lingua e cultura nella Sardegna bizantina. Testimonianze linguistiche dell'influsso greco, Sassari, 1983, pp. 22-24 e P.G. SPANU, La Sardegna bizantina tra VI e VII secolo, Oristano, 1998, p. 126. 
Perché diciamo questo? Perché l'obiezione primaria mossa alla presenza nell' isola del ceto dei $\mathrm{K} \alpha \beta \alpha \lambda \lambda \dot{\alpha} \rho$ ıo consiste nel fatto che insorgono problemi di datazione che, se risolti positivamente, spingerebbero in via di ipotesi a intensificare i legami tra la Sardegna e il mondo greco-orientale intorno al IX e X secolo. Periodo per il quale gli storici ipotizzano un distacco già consistente - per non dire definitivo e irreversibile- dell'isola dall'Impero ${ }^{19}$.

Da un punto di vista culturale - nel senso più lato che si può dare a questo termine - la Sardegna si distaccò in via definitiva dal mondo bizantino solo nel corso dell'XI secolo e da lì in poi entrò in un'orbita italica e europea occidentale, mediante l'avvio delle relazioni con il Papato e le Repubbliche marinare di Genova e Pisa, oltre che, con il passar del tempo, anche con l'Impero germanico e altre compagini statuali europee.

Questi nuovi contatti produssero ovvie influenze anche nel settore del quale ci stiamo occupando nel presente lavoro. Nelle fonti documentarie e iconografiche giudicali vediamo infatti comparire nelle società sarde del Basso Medioevo diversi elementi su cui dobbiamo soffermarci, che testimoniano l'ingresso in esse della cultura cavalleresca nel senso forse più tradizionale del termine. A questo proposito, infatti, va detto che, mettendo da parte il

${ }^{19}$ Proprio di recente Salvatore Cosentino si dimostra incerto dinanzi all'interpretazione di $\kappa \alpha \beta \alpha \lambda \lambda \dot{\alpha} \rho \iota \varsigma$ data da Giulio Paulis per diversi motivi: innanzitutto, perché le terre militari ( $\tau \rho \alpha \tau \imath \tau \tau \iota \kappa \grave{\alpha})$ sono attestate esplicitamente per la prima volta in una Novella di Costantino VII

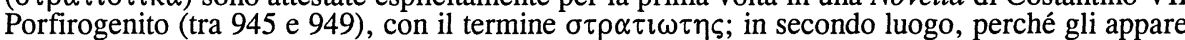
improbabile che la diffusione di $\kappa \alpha \beta \alpha \lambda \lambda \dot{\alpha} \rho \iota \varsigma$ possa essere ricollegata all'installazione di limitanei, perché non risulta che fossero composti da cavalleria. Mentre invece il vocabolo $\kappa \alpha \beta \alpha \lambda \lambda \dot{\alpha} \rho 10 \varsigma$ in greco bizantino è un termine tecnico per indicare il cavaliere almeno dal VI secolo; nella lingua scritta la prima menzione di $\kappa \alpha \beta \alpha \lambda \lambda \dot{\alpha} \rho \mathrm{\rho} \varsigma$ è nel cosiddetto Chronicon Paschale, composto da un ecclesiastico attivo durante il regno del basileus Eraclio I, mentre $\kappa \alpha \beta \alpha \lambda \lambda \dot{\alpha} \rho \eta \varsigma$ compare per la prima volta nei T $\alpha \kappa \tau \imath \kappa \grave{~ d i ~ L e o n e ~ V I ~(886-912) . ~}$

Sempre in merito al tema della cavalleria, F. CARDINI, Alle radici della cavalleria medievale, Firenze, 1997, pp. 245-254 ha messo in rilievo che anche nell'Impero bizantino fin dal VI secolo il ruolo avuto dalla cavalleria pesante, composta anche da Germani, Unni, Balcanici, Anatolici e Armeni, fu notevole, anche se la fanteria -pesante anch'essa- non aveva certo un'importanza secondaria. Va ricordato, inoltre, che al lato di questa prima cavalleria ne operava anche una seconda più leggera, che fungeva da complemento della prima in tutte le circostanze in cui quella non poteva essere impiegata con successo. L'importanza della cavalleria catafratta crebbe ulteriormente nel corso del VII secolo, quando inizió a porsi per l'Impero il problema arabo. In questo periodo la cavalleria aveva già preso il sopravvento sulla fanteria. In aggiunta a questi dati, si potrebbe considerare l'ipotesi avanzata sempre da S. COSENTINO, Potere e istituzioni nella Sardegna bizantina, in Ai confini dell'impero. Storia, arte e archeologia della Sardegna bizantina (P. CORRIAS e S. COSENTINO, ed.), Cagliari, 2002, pp. 7-8, secondo il quale le truppe a cavallo imperiali poterono essere state trasferite nell'isola dall'Africa o dall'Egitto, nella seconda metà del VII secolo, dopo la perdita per l'Impero del litorale nordafricano. E che a partire da questo periodo si fossero diffuse un po' in tutto il territorio lasciando così traccia di esse nei toponimi kaddaris/quaddaris. 
problema di una più esatta definizione dei liberi ab equo come combattenti a cavallo, intendiamo analizzare alcuni brani della non abbondante documentazione che ci permettono di avanzare alcune ipotesi interpretative.

Così facendo ci ricolleghiamo a quanto abbiamo già detto in un nostro recente lavoro a proposito di alcune di queste tracce che ci rimandano alla presenza di cultura cavalleresca nel giudicato di Arborea nella seconda metà del XIV secolo.

Ecco gli elementi in questione.

Il 14 aprile 1392 nell'ambito del processo che i sovrani aragonesi avevano avviato contro i giudici arborensi accusati di ribellione, Joan Fuster soldato di Longosardo rilasciò una testimonianza che si rivela estremamente interessante a proposito del tentativo di Brancaleone Doria di impadronirsi della villa e della fortezza posta nell'estremità nord della Sardegna, giusto dinanzi alla colonia genovese di Bonifacio in Corsica. Negli atti leggiamo infatti che un tale, Bonifacio, fu inviato al detto Fuster dal conte di Monteleone per chiedergli di aiutare i giudicali a entrare a Longosardo; in cambio egli assicurò al soldato iberico che micer Brancha vos farà lo millor hom de Serdenya e us farà cavaller ${ }^{20}$. Testimonianza molto interessante, in quanto vi compare la concessione del cavalierato usata come una sorta di moneta di scambio pregiata.

Altrettanto stimolante per noi si rivela anche la seconda parte della deposizione del soldato aragonese, dalla quale trapela che non solo il Doria aveva deciso di utilizzare la concessione di questo titolo come un incentivo per procurarsi la fedeltà dei suoi interlocutori, ma che in precedenza lo aveva già fatto un imprecisato giudice $a \cdot n$ Roger de Pinòs, que li donà lo castell de Bon Vehí. Dovette trattarsi con ogni probabilità di Mariano IV, che nel 1333 era stato creato cavaliere con tanto di cingolo militare dal re aragonese Alfonso il Benigno. Investitura quanto mai importante, in quanto si credeva che avendola ricevuta da un signore di condizione particolarmente elevata il neocavaliere sarebbe stato reso partecipe dell'onore e della dignità di colui che lo creava $^{21}$.

\footnotetext{
${ }^{20} \mathrm{ACA}, \mathrm{C}$, Procesos contra los Arborea, vol. X, ff. 123r-126r.

${ }^{21}$ Per la verità, sempre Mariano IV risulta coinvolto in un altro episodio che possiamo riferire al tema di cui ci stiamo occupando. In un documento conservato presso l'Archivio di Stato di Pisa, Diplomatico Cappelli, 1385, citato da S. PETRUCCI, Re in Sardegna, a Pisa cittadini. Ricerche sui «dominii Sardinee» pisani, Bologna, 1988, p. 143 e nota 30 , è contenuta l'infeudazione della villa di Turri da parte del giudice a Pietro Gualandi, definito «militem ipsius domini iudicis». Questa espressione non consente di affermare con sicurezza che il Gualandi citato

«Anuario de Estudios Medievales», 33/2 (2003), pp. 849-879 .- ISSN 0066-5061.
} 
Tornando al Doria, tutta la questione venne presentata dall'emissario del conte di Monteleone come una buona occasione di ascesa sociale per Joan Fuster, il quale avrebbe potuto accordarsi con il Doria (ho podrets mercadeiar) e sistemarsi (vos farà lo millor hom de Serdenya), anche perché dal resoconto del testimone si evince che egli non doveva godere di una buona condizione economica: no havets diner ni malla et mal recapte no us manca.

La presenza di questa testimonianza, con i suoi riferimenti all'uso del cavalierato nella Sardegna giudicale, ci ha spinto a proseguire con la ricerca di altri riferimenti documentari che fornissero nuovi elementi sulla società militare presenti nello stato indigeno alla fine del Medio Evo.

$E$ in effetti alcuni dati interessanti in tal senso emergono da fonti narrative sarde che non riguardano solo il Giudicato di Arborea e il XIV secolo. Una - nel dettaglio, il Libellus Iudicum Turritanorum- si riferisce al Giudicato di Logudoro e alle vicende svoltesi alla metà del XII secolo. La testimonianza in questione si riferisce al giudice Gonnario di Torres (1116post 1153), una figura tra le più significative di tutta la storia giudicale sarda. Costui, dopo aver eliminato gli esponenti di una famiglia politicamente a lui contraria, in ricompensa dell'aiuto che aveva ricevuto contro i suoi nemici da Ittocorre Gambella, suo tutore, lu fetit cavalleri et li donait sas villas detsa Turpe de Romangia, cun sos saltos et terras [il grassetto è nostro] ${ }^{22}$. In questo caso la concessione del titolo cavalleresco è accompagnata dalla donazione di territori appartenuti al demanio statale ${ }^{23}$.

fosse stato armato cavaliere da Mariano IV, e non fosse piuttosto solo un miles al servizio del suo dominus, esattamente come avveniva in contemporanea in altre parti d'Europa. Non si può escludere, tuttavia, che a concedere la militia al giovane Gualandi fosse stato il giudice di Arborea, in quanto la famiglia toscana prestava servizio presso la corte giudicale di Oristano fin dalla metà del XIII secolo, in considerazione del prestigio che godeva Mariano IV come cavaliere armato col cingolo militare dal re aragonese Alfonso il Benigno e come sovrano arborense. In merito alla datazione di questo interessante documento, Petrucci evidenzia come non sia possibile essere precisi a causa del pessimo stato di conservazione della pergamena. Sulle cerimonie di investiture cfr. tra gli altri M. KEEN, La cavalleria, Napoli, 1986, p. 124 e J. FLORI, L'essor de la chevalerie. XI $-X I I^{e}$ siècles, Genève, 1986.

${ }^{22}$ Libellus Judicum turritanorum, p. 48. Gonnario di Torres, ancora minorenne alla morte del giudice Costantino, fu portato a Pisa da Ittocorre Gambella per sfuggire ai pericoli di morte rappresentati dai membri della famiglia Athen, imparentati con un aspirante al trono giudicale logudorese, Saltaro, che forse era un fratellastro di Gonnario.

${ }^{23}$ Esempi di simili donazioni di villaggi e terreni fatte dai giudici sardi a isolani o anche a continentali, fossero essi singole persone o Ordini religiosi, hanno fatto sì che diversi studiosi ipotizzassero la presenza di strutture feudali in Sardegna anche prima dell'arrivo degli Aragonesi. $\mathrm{Su}$ questo dibattuto tema, anche per la sintesi delle posizioni di precedenti studiosi cfr. $\mathrm{M}$. TANGHERONI, La Sardegna prearagonese: una società senza feudalesimo?, in ID., Sardegna mediterranea, Fonti e studi del Corpus membranarum italicarum. I serie, Studi e ricerche, XXIII, Roma, 1983, pp. 57-84. 
Forse possiamo leggere questo episodio come un esempio di accollata cavalleresca, che non era niente altro che un modo universalmente riconosciuto per onorare e ricompensare il coraggio mostrato nel campo di battaglia ${ }^{24}$. Nel nostro caso, più che altro, da parte del giudice turritano si trattava di ricompensare una fedeltà che gli aveva permesso di avere salva la vita in un primo momento, e di poter giungere al trono giudicale a distanza di anni eliminando i membri di una potente famiglia di maiorales che gli contendeva il governo del Logudoro

Il caso di Gonnario che abbiamo appena analizzato precede i seguenti non solo da un punto di vista cronologico, ma anche perché essi hanno in comune un altro elemento. Il giudice di Torres e i protagonisti delle altre testimonianze che esamineremo di seguito sono strettamente legati all'area toscana non solo su un piano politico.

In questo senso, un'altra fonte che contiene dati interessanti per il discorso che cerchiamo di sviluppare è una cronaca di un anonimo autore pisano che raccontò gli avvenimenti che videro protagonista la città dell' Arno in un arco di tempo compreso tra il 1231 e il $1295^{25}$. Al suo interno sono contenute alcune indicazioni che si rivelano estremamente utili per il nostro tema. Il cronista, infatti, a proposito degli avvenimenti che portarono alla caduta della capitale del Giudicato di Calari nel 1258, raccontò che il giudice di Gallura, insieme a diversi personaggi di spicco di Pisa e al giudice di Arborea Guglielmo, misero l'assedio alla città di Santa Gilla e a Castel di Castro, il nuovo nucleo abitativo edificato dai Visconti nel 1216. Dopo la vittoria il giundici del Galuri fe' cavaglieri el conte Gerardo de Donoraticho in sul corpo del marchese $e^{26}$. Questo brano merita di essere commentato nel dettaglio. Iniziamo dai personaggi che vi compaiono, perché questo può aiutarci a comprendere un po' di più questo innesto di cultura cavalleresca in Sardegna. Il giudice di Gallura menzionato era Giovanni Visconti, figlio del podestà di Pisa e di Contessa di Capraia, zia del giudice di Arborea Guglielmo di Capraia, che resse quel trono dal 1250 alla sua morte nel 1264. Il marchese citato nel documento, sul cui cadavere avvenne la cerimonia di conferimento del cavalierato era Guglielmo III-Salusio VI giudice di Calari, marchese di

\footnotetext{
${ }^{24}$ M. KEEN, La cavalleria, p. 265.

${ }^{25} \mathrm{E}$. CRISTIANI, Gli avvenimenti pisani del periodo ugoliniano in una cronaca inedita, in “Bollettino Storico Pisano", XXVI (1957), pp. 3-104.

${ }^{26} \mathrm{E}$. CRISTIANI, Gli avvenimenti pisani, p. 55.

«Anuario de Estudios Medievales», 33/2 (2003), pp. 849-879 .- ISSN 0066-5061.
} 
Massa. Il beneficiato del titolo di cavaliere fu il conte Gherardo Donoratico Della Gherardesca, zio del più noto Ugolino reso immortale da Dante ${ }^{27}$.

Come si può notare fin da una prima occhiata si tratta in tutti i casi di persone di origine e cultura toscana che a diverso titolo si trovarono ad agire in Sardegna, ma che in ogni caso mantennero legami di diversa natura con la regione di provenienza. Sembra quasi di muoversi in un contesto generale italiano, abituato alla presenza di cavalieri di professione non solo di origine peninsulare ma anche di altre regioni europee. In effetti, questa che abbiamo appena commentato non è la sola attestazione in merito al tema del cavalierato che riporta la anonima cronaca pisana. Facendo un balzo in avanti di circa vent'anni, la nostra fonte cita un altro episodio della storia giudicale interessante per noi: un attacco condotto nel 1274 dal giudice di Arborea Mariano II contro Castel di Castro, in occasione del quale vi fu uno scontro con il giudice di Gallura Giovanni Visconti che venne duramente sconfitto. Anche allora, in seguito alla battaglia, lo iudici d'Arborea fece cavaglieri li quali furno questi: messere Cino Villano, messere Villano, messere Giano Chitulo, messere Betto Nazari, messere Puccio Lanbatto de'Lanfranchi, messere Gano Scornigiano, messere Puccio di Casalei et altrt ${ }^{28}$.

A differenza di quanto notavamo prima a proposito di Gonnario di Torres, questo di Mariano II appare come un classico esempio di accollata cavalleresca, anche se va evidenziato che questa modalità di concessione del cavalierato era molto meno comune di quella che avveniva alla vigilia della battaglia, quando esso era attribuito per infondere ancor più coraggio ed energia nei combattenti ${ }^{29}$.

A parte la testimonianza del Libellus riferita alla metà del XII secolo, i dati sui rapporti di diversa natura intrattenuti da Mariano II di Arborea con l'area toscana hanno spinto qualche studioso a sostenere che la storia sarda della seconda metà del Duecento «sia stata vissuta come un'epopea cavalleres-

\footnotetext{
${ }^{27}$ Per una più precisa collocazione di tutti i personaggi compresi nella citazione delle cronaca pisana rimandiamo a Genealogie medioevali di Sardegna (L.L. BROOK, F.C. CASULA, M.M. COSTA et alii, ed.), Cagliari-Sassari, 1984, Tav. XVII, Lemma 10 per Giovanni Visconti giudice di Gallura; Tav. X, Lemma 18 per Guglielmo di Capraria giudice di Arborea; Tav. XXVIII, Lemma 19 per Guglielmo III-Salusio VI di Cepola, marchese di Massa e giudice di Calari e, infine, Tav. XI, Lemma 8 per il conte Gherardo della Gherardesca. Su Ugolino della Gherardesca e la sua morte nella "torre della fame" cfr. D. AlighIERI, La Divina Commedia, Inferno, canto XXXIII, vv. 1-90.

${ }^{28}$ E. CRISTIANI, Gli avvenimenti pisani, p. 75. Su Mariano II di Arborea rimandiano a Genealogie medioevali di Sardegna, Tav. XXII, Lemma 6.

${ }^{29}$ M. KEEN, La cavalleria, p. 265.

«Anuario de Estudios Medievales», 33/2 (2003), pp. 849-879 .- ISSN 0066-5061.
} 
ca da molti dei suoi protagonisti», anche se va aggiunto che, per quanto si intravede dalle fonti, questi protagonisti sono tutti o quasi personaggi di origine toscana. Il che ci porta a ipotizzare che presumibilmente furono loro stessi o alcuni consimili a portare con sé questa cultura nell'isola ${ }^{30}$.

Un altro ambiente di provenienza di questa cultura cavalleresca e cortese può essere senz'altro considerata la corte di Barcellona. Sono ben noti i contatti plurisecolari tra la dinastia dei conti-re catalani e i giudici di Arborea nello specifico; ai fini del nostro discorso, due furono i momenti cruciali di queste relazioni: l'epoca di Barisone I d'Arborea (1146-1185) che si sposò con Agalbursa, cugina del re d'Aragona Alfonso I; e la prima metà del XIV secolo, dall'arrivo dei Catalano-Aragonesi in Sardegna all'inizio delle ostilità con gli Arborensi ${ }^{31}$.

Altri elementi da considerare con attenzione nel tentativo di valutare con maggiore precisione la reale portata degli influssi culturali catalani nell'isola e, nella fattispecie, la presenza di milites nella Sardegna giudicale prima dell'arrivo degli Aragonesi, potrebbero provenire dalla sfragistica, alla quale varrebbe la pena di dedicare ulteriori ricerche. Già in altri lavori ci siamo soffermati sulle tipologie di sigilli usati dai giudici d'Arborea nel XII e XIII secolo, e notavamo che esse intorno alla fine del XII secolo hanno subito una variazione che riguarda da vicino il tema di cui stiamo trattando ${ }^{32}$.

In questo contesto, un vero e proprio spartiacque dev'essere considerato il 1186, in quanto fino ad allora nel Giudicato arborense si usavano ancora sigilli plumbei di chiara matrice e caratteristiche tipologiche bizantine; mentre invece, a partire da quella data fece la sua comparsa il sigillo di Ugone I che sembra appartenere alla tipologia equestre, recando nel

${ }^{30}$ Memoria de las cosas, p. XLIX.

${ }^{31} \mathrm{P}$. MANINCHEDDA, Sui rapporti tra la poesia popolare sarda e la tradizione lirica provenzale e catalana, in ID., Studi catalani e provenzali, Cagliari, 1996, pp. 50-55. Il fratello di Agalbursa, il visconte di Bas, aveva stretti rapporti con la città di Besalú da cui proveniva il poeta Ramon Vidal, autore di celebri Razos de Trobar, di cui fu composta in Sardegna alla fine del Duecento una versione in versi da Terramagnino da Pisa: la Doctrina d'Acort. Egli utilizzò nella Sardegna sardo-pisana il modello catalano dei Razos, senza alcuna semplificazione dei concetti e dell'impianto, il che appare indicativo delle caratteristiche del suo pubblico non digiuno della poesia provenzale. Pubblico col quale si confrontarono anche i Catalani. A questi dati Maninchedda aggiunge anche che nel XIV secolo Timbora de Rocabertí, moglie del giudice Mariano IV d'Arborea, era sorella di Dalmau, abate di Vilabertran, a cui era dedicato il Compendi de la conexença dels vicis del Gai Saber di Joan de Castelnou, piccola sintesi delle Ley d'amor. In considerazione di tutti gli elementi noti, il filologo sardo propende per il XIV secolo come periodo di maggior influenza della cultura poetica catalana e provenzale su quella sarda.

${ }^{32}$ L. GALlINARI, Gli ultimi anni di esistenza, passim. 
verso un cavaliere mentre nel recto vi era la figura di un sovrano assiso in trono con il braccio destro che impugnava una spada sguainata ${ }^{33}$.

$\mathrm{Da}$ questo momento nei sigilli arborensi la tipologia equestre fu contemplata regolarmente, il che non pare casuale se si considera che il giudice Ugone I era in realtà il figlio di Sinispella de Lacon-Serra - figlia del precedente giudice Barisone I- e di Ug Ponç de Cervera, visconte di Bas. Non sembra quindi fortuito che questa tipologia di sigilli che mostra più di una caratteristica in comune con quelle coeve dei sovrani aragonesi, penetrasse in Arborea proprio grazie a questa famiglia che proveniva dalla Catalogna, una delle aree maggiormente feudalizzate di tutta l'Europa mediterranea $^{34}$.

Detto questo, dunque, dagli elementi in nostro possesso sembrerebbe possibile sostenere che anche le società giudicali parteciparono alla cultura cavalleresca almeno dalla metà del XII secolo, anche se non si può escludere che ciò avvenisse già da prima. Da quando cioè, nell'XI, secolo l'isola fuoriuscì in via definitiva da un ambito culturale bizantino per "entrare" -pur con le dovute precisazioni che in questa sede sarebbe fuorviante fare- nel mondo europeo occidentale. Questa ipotesi, ci pone in realtà alcuni interrogativi ai quali non possiamo dare risposte certe ed esaurienti. In cambio, possiamo proporre alcune ipotesi interpretative.

Il principale quesito che ci poniamo da diverso tempo riguarda l'eventuale presenza nella società giudicale arborense tra il XIV e il XV secolo di un ceto di cavalieri di professione, di milites equiparabili agli altri che si riscontrano in altre aree europee. $E$ anche le fonti appena commentate, pur nella loro apparente chiarezza, in realtà non rispondono a tutte le nostre domande. Infatti, se già alla metà del Dodicesimo secolo risulta attestata la presenza di cavalieri, creati dagli stessi giudici, appare quanto meno strano che nelle fonti successive essi non compaiano pressoché mai.

Sempre in merito al problema dell'esistenza della cavalleria nel giudicato e delle sue caratteristiche, un altro dato su cui vale la pena di soffermarsi ci è offerto dalla Carta de Logu arborense, il principale testo

\footnotetext{
${ }^{33} \mathrm{Per}$ la ricostruzione dei sigilli dei giudici arborensi rimandiamo a F.C. CASULA, Influenze catalane nella cancelleria giudicale arborense del sec. XII: $i$ sigilli, in F.C. CASULA-L. D'ARIENZO, Studi di paleografia e diplomatica, Padova, 1974, pp. 116-117. Il documento in cui appare menzionato il sigillo di Ugone I è in P. TolA, Codex Diplomaticus Sardiniae, Tomo I, Parte I, Torino, 1861, doc. CXVII, pp. 256-257.

${ }^{34} \mathrm{Su}$ Ug Ponç de Cervera, giudice con il nome di Ugone I vedasi F.C. CASUlA, Dizionario storico sardo, Sassari, 2002, ad vocem "Ugone I, re di Arborea".
} 
giuridico della civiltà giudicale che a tutt'oggi possediamo. Il capitolo XCI che tratta dei liberi ab equo (lieros de cavallu in sardo arborense) imponeva che essi dovessero tenere un cavallo del valore superiore alle dieci lire et tota arma qui bisongiat ad homjni de couallo at sa sardisca ${ }^{35}$.

L'intepretazione di questo breve brano potrebbe non essere del tutto univoca ma duplice. Nel senso che, in un caso, l'espressione at sa sardisca sembrerebbe doversi riferire alle armi di cui si sarebbe dovuto dotare il cavaliere; ebbene, il semplice fatto che nel testo esse venissero indicate così parrebbe indicare che avessero caratteristiche peculiari che le individuavano come sarde e che, nel contempo, la differenziavano dalle altre armi che si usavano un po' ovunque nei campi di battaglia coevi. Se questa prima interpretazione fosse la corretta, è possibile che il legislatore intendesse qui fare riferimento anche alla più tipica delle armi medievali sarde: la virga.

Questo almeno secondo il testo contenuto nell'edizione manoscritta quattrocentesca. L'edizione del 1567, tradotta in italiano da Giovanni Maria Mameli de' Mannelli nel 1805 presenta oltre a differenze linguistiche, in questa sede non importanti, anche una variante che invece ci riguarda da vicino $^{36}$. Il testo, infatti, recita così: totu armadura chi bisongiat ad homini de cavallu, assa sardisca [il grassetto è nostro]. La differenza, tra le due lezioni, com'è immediatamente comprensibile, non è ininfluente in quanto in questo secondo caso bisognerebbe ammettere che i cavalieri sardi indossassero corazze. Anche per queste ultime vale quanto dicevamo in precedenza, in quanto è possibile che esse avessero caratteristiche particolari che permettevano di distinguere gli homjni de couallo at sa sardisca dai cavalieri tout-court presenti in tutta Europa.

Purtroppo, anche in questo frangente dobbiamo lamentare l'abituale carenza di fonti; soprattutto, nella fattispecie, di tipo iconografico che ci avrebbero potuto fornire qualche elemento di valutazione in più.

Nel secondo caso a cui ci riferivamo in precedenza, invece, l'espressione at sa sardisca potrebbe anche riferirsi all'homjni de couallo, il quale, per essere così connotato, avrebbe indubbiamente dovuto avere qualche

\footnotetext{
${ }^{35}$ Per il testo quattrocentesco del Codice cfr. E. BeSTA-F.E. GuARNERIO, Carta de Logu de Arborea, in "Studi Sassaresi", III (1905), p. 43.

${ }^{36} \mathrm{Sul}$ problema delle diverse edizioni del principale testo giuridico arborense cfr. F.C. CASula, La 'Carta de Logu' del regno di Arborèa, Cagliari, 1994, pp 26-27, e per la lezione dell'edizione del 1567, p. 118. 
elemento peculiare per distinguerlo dagli altri combattenti a cavallo presenti nei territori giudicali, spesso assoldati dagli stessi signori arborensi.

In mancanza di altri dati certi si possono solo avanzare ipotesi. In linea di massima, l'impressione che ci stiamo formando con il procedere delle ricerche è che nella Sardegna giudicale dei secoli XIV e XV accanto a una cavalleria pesante tradizionale -composta perlopiù da elementi estranei all'isola, pur con qualche eccezione ${ }^{37}-$ vi dovesse essere una cavalleria indigena composta dai liberi ab equo o homjnis de couallu che, come osservavamo in precedenza, non era omologabile alla prima. Forse, e sottolineiamo forse, questa seconda cavalleria aveva caratteristiche che potevano assimilarla ad altre omologhe sviluppatesi nel bacino del Mediterraneo. Ci viene in mente la caballería villana. Si trattava di una cavalleria più mobile e armata in modo più leggero rispetto alle coeve cavallerie del Nord Europa - portava una corazza di strisce di cuoio o feltro rinforzate a volte da lamine di metallo come usavano anche i Mori-sviluppatasi nella Penisola iberica cristiana dal X secolo. Essa era composta da piccoli proprietari e da uomini liberi enfiteuti delle ville del Duero, legati al territorio da difendere dai Musulmani, i quali si facevano carico di mantenere a proprie spese cavallo e armi necessarie e che avrebbero prestato servizio in guerra ${ }^{38}$. Il parallelismo con i lieros de cavallu ci viene proposto dall'articolo XCI della Carta de Logu che abbiamo menzionato in precedenza. In esso, infatti, la legislatrice arborense dispose che i lieros de cavallu arborensi, anch'essi uomini liberi, siant semper apparizzados cun sos dittos cavallos ed armas pro fagheri sa mostra, e pro cavalcari quando Nos illos fagheremos recheder (siano sempre pronti con i detti cavalli e le armi alle rassegne e alle cavalcate quando glielo faremo richiedere $)^{39}$.

\footnotetext{
${ }^{37}$ Vedasi la testimonianza di Antonio Correja il quale tra i comandanti dell'esercito giudicale di Mariano IV menzionò quidam miles vocatus dominus Iohannes Datzori. L'attribuzione della militia a questo personaggio dovrebbe sottintendere che Giovanni de Atzori dovesse essere in grado di mantenere economicamente almeno un cavallo da guerra e un'armatura confacente a chi portava un titolo simile. ACA, C, Procesos contra los Arborea, vol. V, f. $81 \mathrm{r}$.

${ }^{38} \mathrm{Su}$ questo tema delle cavallerie mediterranee e delle loro caratteristiche tipologiche nei confronti di quelle del Nord Europa rimandiamo a F. CARDINI, Alle radici della cavalleria, pp. 286 n. 92, 326-329 e alla bibliografia ivi contenuta e a PH. CONTAMINE, La guerre au Moyen Áge, Paris, 1980 , pp. 245246 che cita anche gli hobelars inglesi, le gens de cheval francesi, sottolineando che fu soprattutto nella periferia meridionale e orientale dell'Europa che la cavalleria leggera ebbe un ruolo tattico ben determinato.

${ }^{39}$ F.C. CASUlA, La “Carta de Logu”, p. 118.
}

«Anuario de Estudios Medievales», 33/2 (2003), pp. 849-879 . - ISSN 0066-5061. 
Se questa ipotesi interpretativa ha un qualche fondamento, ciò potrebbe spiegare perché i sardi giudicali ancora nei primi anni del Quattrocento erano soliti iniziare i propri scontri con le truppe di terra, dal momento che una cavalleria con le caratteristiche come quelle descritte in precedenza non avrebbe potuto reggere un urto frontale con una cavalleria pesante. Questo, infatti, è quanto sembra ricavarsi dalla testimonianza dall'annalista aragonese Jerónimo Zurita in merito alla battaglia di Sanluri, lo scontro campale che nel giugno 1409 oppose i sardi arborensi ai catalano-aragones ${ }^{-40}$.

In merito a questo fondamentale evento bellico il cronista iberico ci fornisce due informazioni che si rivelano di primario interesse ai fini del nostro tentativo di ricostruzione. Egli infatti da un lato riportò che se había dado tal orden por el rey que quinientos de caballo de los que llamaban bacinetes de la gente más escogida y de los más señalados caballeros se pusiesen a pie si los sardos echasen delante sus peones como era su costumbre [il grassetto è nostro]. Di seguito, però, aggiunse anche che dopo aver avvistato le truppe giudicali poste su una collina nei pressi del centro abitato di Sanluri, Martino il Giovane dispose la cavalleria alla sua destra e la fanteria alla sinistra. Dopo di che comenzóse la batalla muy furiosamente en los primeros encuentros entre la caballería del rey y la de los enemigos, $y$ en ella fueron a tierra muchos caballeros sardos [...].

Riassumendo, dunque, ecco i dati forniti da Zurita: 1) agli inizi del $\mathrm{XV}$ secolo i sardi giudicali erano soliti iniziare le battaglie con le truppe di terra; 2) in occasione della battaglia di Sanluri, però, ad avviare le ostilità furono la cavalleria iberica e quella sarda, anche se bisognerebbe intendersi bene sul significato da dare a questi due termini.

Perché proprio in questa occasione -che i partecipanti dovettero avvertire come fondamentale per la propria causa, se non altro per l'elevato numero di soldati coinvolti; per la presenza del re di Sicilia nello schieramento iberico e del giudice d'Arborea nell'altro - i giudicali cambiarono tecnica di combattimento? Una plausibile risposta affermativa può essere rappresentata da Guglielmo II visconte di Narbona e sovrano arborense, formatosi nei campi di battaglia francesi ove da oltre mezzo secolo si combattevano gli Inglesi nella guerra dei Cent'Anni. Egli, probabilmente, volle combattere come sapeva fare. Se ritorniamo ancora una volta alla citazione di Zurita, infatti, osserviamo che egli riporta come il visconte uscì da Sanluri per porre il suo

${ }^{40} \mathrm{~J}$. ZURITA, Anales de la Corona de Aragón (A. CANELlas LóPEZ, ed.), vol. 4, Zaragoza, 1978, libro X, p. 915.

«Anuario de Estudios Medievales», 33/2 (2003), pp. 849-879 .- ISSN 0066-5061. 
esercito al riparo di una collina ove non venne notato dalle spie iberiche ${ }^{41}$. Da questa altura, poi, le truppe giudicali sferrarono l'attacco iniziando con la cavalleria, secondo i dettami tradizionali delle battaglie campali dell'epoca.

Il riferimento alla cavalleria sarda ci spinge a porci un altro interrogativo: si trattava di semplici combattenti a cavallo, da non identificare con i milites, per intenderci, e da considerare liberi ab equo come quelli che abbiamo visti citati nei Procesos di metà Trecento? Oppure, erano veri e propri cavalieri arruolati dal giudice forse nelle regioni francesi vicine ai suoi dominî, che egli potrebbe aver assoldato al fine di rinforzare l'esercito giudicale in previsione di una battaglia frontale in cui l'elemento risolutivo era senz'altro costituito ancora dalla cavalleria?

È difficile se non impossibile fornire un'interpretazione decisiva su questi avvenimenti perché purtroppo la citazione di Zurita non aggiunge ulteriori dettagli che possano far propendere per una soluzione definitiva.

$\mathrm{Su}$ un punto però intendiamo soffermarci ancora, poichè ci permette di riallacciarci con quanto dicevamo in precedenza in merito alla diffusione nelle società giudicali della milizia. Ciò che risulta interessante notare in questa sede è che, pur considerando le testimonianze documentarie risalenti alla metà del XIII secolo, che citavano la presenza di milites in Arborea, Logudoro e Gallura, di fatto agli inizi del Quattrocento gli eserciti giudicali -almeno a detta di Zurita - erano soliti iniziare le battaglie campali con la fanteria, affidando a questa l'onere del primo scontro che in molti casi poteva risultare decisivo per il successivo andamento della battaglia ${ }^{42}$. A parer nostro risulta significativo che si fosse sviluppata questa tattica di combattimento, forse dovuta all'esiguità della cavalleria giudicale e al suo armamento. Sembrerebbe altrimenti strano che in un periodo di circa due secoli -caratterizzato da guerre pressoché continue tra i Giudicati prima e tra l'Arborea e l'Aragona poi- si relegasse a un ruolo secondario proprio la cavalleria che normalmente costituiva in tutta l'europa occidentale il corpo d'élite degli eserciti medioevali. In questo senso, quindi, la "originalità"

\footnotetext{
${ }^{41} \mathrm{La}$ manovra di Guglielmo II di far uscire il grosso dell'esercito giudicale dalla villa di Sanluri non era sbagliata in quanto Martino il Giovane aveva deciso di andare ad assediare la suddetta villa, forte di un esercito di circa 11.000 uomini. Abbiamo fatto le nostre considerazioni su questi eventi in L. GALLINARI, Gli ultimi anni di esistenza, in corso di stampa.

${ }^{42} \mathrm{~A}$ proposito della stranezza della tattica di guerra impiegata abitualmente dai Giudicali, secondo la testimonianza di Zurita, va detto che PH. ConTAMINE, La guerre au Moyen Âge, pp. 250-257 notava che tra la metà del Trecento e quella del Quattrocento la fanteria attraversò un periodo di decadimento quantitativo e qualitativo, venendo, per di più, sostituita da balestrieri, tiratori e pavesai.

«Anuario de Estudios Medievales», 33/2 (2003), pp. 849-879 .- ISSN 0066-5061.
} 
giudicale sarebbe stata superata proprio dal visconte di Narbona, il quale apparteneva a una cultura militare diversa, incentrata sul ruolo fondamentale della militia.

Una conferma di questa nostra interpretazione potrebbe venire da alcuni brani di una fonte che abbiamo già citato in precedenza. Riferendosi a un evento bellico prodottosi nel 1478 - la battaglia di Macomer tra Leonardo de Alagón, ultimo discendente degli Arborea, e il viceré Nicolau Carróz-, il cronista segnala che lo stesso señor marqués [Leonardo de Alagón]...cavalgó en un cavallo blanco a la bastarda e che vi erano altri quinze $o$ veinte ombres de armas blancas, sin otros muchos a la bastarda ginetes sardos, según su acostumbrado cavalgar [il grassetto è nostro]. In questo contesto risulta estremamente significativo l'uso del termine ginete, che indica un soldato appartenente alla cavalleria leggera, abbinato all'aggettivo acostumbrado che ci permette di ribadire, con una certa sicurezza in più, quanto già dicevamo in precedenza in merito alle caratteristiche della cavalleria giudicale anche dei secoli precedenti ${ }^{43}$

Questa “originalità" giudicale comporterebbe anche un'altra conseguenza. La letteratura cortese a cui abbiamo accennato in precedenza in realtà non avrebbe influenzato più di tanto le società sarde. Nel senso che, pur accettando quanto sosteneva Maninchedda -in merito agli elementi cortesi e cavallereschi di alcuni personaggi quali Mariano II di Arborea- essa sarebbe dunque rimasta al livello di puro modello artistico, conosciuto e apprezzato nelle corti giudicali che ospitavano persone di diversa provenienza geografica e culturale. Probabilmente, però, non dovette riuscire ad avere significativi riflessi nel campo delle attività belliche sarde, almeno secondo quanto ci riportano le fonti finora conosciute. Ma ciò non significa che le società giudicali rimasero del tutto insensibili al fascino esercitato dal mondo cavalleresco-cortese. Infatti, alle fonti esaminate in precedenza, possiamo aggiungerne anche altre che ci permettono di osservare alcuni riflessi di questo complesso mondo nella struttura delle società giudicali in relazione al tema della guerra e dei suoi partecipanti.

Analizzando i Procesos contra los Arborea, infatti, si rinvengono altri indizi che possiamo analizzare un po' dettagliatamente in quanto ci forniscono spunti per ulteriori riflessioni.

${ }^{43}$ Cfr. Memoria de las cosas, p. 54.

«Anuario de Estudios Medievales», 33/2 (2003), pp. 849-879 .- ISSN 0066-5061. 
In primo luogo vogliamo soffermarci su una lunga lettera inviata il 29 novembre 1391 da Brancaleone Doria al governatore generale del Regno di Sardegna Joan de Montbuy ${ }^{44}$. In essa il conte di Monteleone parlava della pace stipulata nel 1388 dal sovrano aragonese Giovanni I con sua moglie Eleonora, giudicessa di Arborea. A proposito delle regioni dell' isola che facevano parte del Regnum Sardiniae e che erano state prima conquistate dai giudicali e quindi restituite al monarca iberico, il Doria disse che lo si era fatto nonostante che aquelles terres eren hagudes e gonyades de bona e justa guerra per la casa d'Arborea, et per los sards sotsmesos de aquella, axí com és clar e manifest a tothom [il grassetto è nostro]. Troviamo significativo il riferimento fatto dal conte di Monteleone al concetto di buona e giusta guerra, che tanta fortuna aveva avuto un po' in tutta l'Europa occidentale del Basso Medioevo. Volendo fare una lettura antropologico-culturale, potrebbe essere ancor più significativo andare alla ricerca di quali erano le basi teoricogiuridiche di questo concetto, che poterono avere interessanti risvolti sulla storia giudicale. È noto che fin all'incirca dalla metà del XII secolo il Decretum di Graziano aveva codificato le quattro condizioni perché si potesse dichiarare una giusta guerra: tra queste, due sembrano adattarsi al nostro contesto storico. Una, la prima, che la guerra fosse dichiarata dal principe; la seconda, che la guerra fosse motivata con il recupero di beni sottratti ${ }^{\text {i5 }}$.

Vediamo il primo punto: che il Doria ritenesse che sua moglie, allora giudicessa-reggente per conto del figlio minore, fosse una principessa a tutti gli effetti lo disse egli stesso molto chiaramente in un'altra sua missiva che in quegli stessi anni indirizzò al governatore generale di Sardegna. A questi sottolineò che l'autorità che, de facto, egli esercitava per la Casa d'Arborea, la doveva a madona Elienor nostra muller, que és jutgessa d'Arborea. Questa autorità derivava alla moglie in quanto filla e succehidora per son pare en lo

${ }^{44}$ ACA, C, Procesos contra los Arborea, vol. IX, ff. 51r-52v.

${ }^{45}$ Comè noto il concetto di guerra giusta affonda le sue radici nella Tarda Antichità, dovendosi far risalire a personalità del IV secolo come sant'Agostino e sant'Ambrogio che, dietro la pressione degli eventi politici del loro tempo, giunsero a legittimare coloro che combattevano per la difesa della pace e della giustizia e contro i popoli barbari visti come oppressori degli indifesi e dei deboli. Su questo interessante concetto che attraversa tutto il Medioevo vedasi tra gli altri Ph. ConTAMINE, La guerre au Moyen Age, pp. 448-462, F. CARDINI, Alle radici della cavalleria, pp. 202-212.

«Anuario de Estudios Medievales», 33/2 (2003), pp. 849-879 .- ISSN 0066-5061. 
Jutgat d'Arborea, la qual Casa d'Arborea ha D Anys que ha hauda senyoria en la present illa, e non la derivava ne de rey ne de regina ${ }^{46}$.

Non sappiamo con certezza se nel Giudicato di Arborea della seconda metà del XIV secolo fossero conosciute le opere di due dei principali giuristi italiani dell'epoca, che si occuparono, tra le altre cose, anche del delicato problema della giusta guerra. Parliamo di Bartolo da Sassoferrato e di Giovanni da Legnano, i quali sottolineavano che nessuno doveva prendere le armi per partecipare a una guerra che non fosse giusta. E secondo loro non rientravano in questa categoria tutte le guerre che fossero state dichiarate da qualcuno che non avesse il rango di principe sovrano ${ }^{47}$. La circostanza che $i$ due giuristi italiani potessero essere conosciuti a Oristano non è del tutto improbabile, considerato che la testimonianza che abbiamo appena citato del Doria è del novembre 1391, di poco precedente quindi la pubblicazione della Carta de Logu da parte della giudicessa Eleonora, la quale fece realizzare dai suoi giuristi una nuova edizione del Codice legislativo del Giudicato che probabilmente fu promulgato nel 1392 . Non sarebbe del tutto illogico pensare che questi giuristi, che dovevano aggiornare il testo legislativo, tenessero conto anche delle ultime interpretazioni del diritto che pressoché in contemporanea i loro più insigni colleghi elaboravano nelle più prestigiose università italiane $^{48}$.

$\mathrm{Nel}$ caso in cui effettivamente i giurisperiti giudicali -e quindi, indirettamente, la famiglia regnante oristanese- avessero conosciuto gli studi su accennati, il richiamo alla guerra giusta fatto da Brancaleone sarebbe stato un ulteriore modo per sottolineare la sovranità della propria consorte agli

${ }^{46}$ ACA, C, Procesos contra los Arborea, vol. X, ff. 141-142v. Questa lunga lettera di Brancaleone Doria è stata pubblicata da F.C. CASULA, Carte reali diplomatiche di Giovanni I il Cacciatore, re d'Aragona, riguardanti l'Italia, Padova, Cedam, 1977, doc. 153, pp. 185-189.

${ }^{47} \mathrm{Su}$ Bartolo da Sassoferrato (1314-1357) e la sua imponente produzione giuridica oggi raccolta in un Corpus di opere di alcune delle quali si contesta la sua paternità e su Giovanni da Legnano, autore del Tractatus de bello, de represaliis et de duello terminato nel 1360, e la cospicua bibliografia sui giuristi italiani preferiamo rimandare a Dizionario Enciclopedico del Medioevo, ad vocem "giuristi italiani".

${ }^{48} \mathrm{~A}$ proposito delle influenze del diritto romano nella Sardegna giudicale F. SINI, Diritto romano nella Carta de Logu d'Arborea: $i$ capitoli "De appellationibus" $e$ "De deseredari", in Giudicato d'Arborea e Marchesato di Oristano: proiezioni mediterranee e aspetti di storia locale, “Atti del I Convegno Internazionale di Studi”, Oristano, 5-8 dicembre 1997, (G. MELE, ed.), vol. II, Oristano, S'Alvure, 2000, pp. 985-986 sostiene che si possano distinguere influssi della Magna Glossa di Accursio ( $\uparrow 1263)$ nell'utilizzo di vari significati del termine respublica.

«Anuario de Estudios Medievales», 33/2 (2003), pp. 849-879 .- ISSN 0066-5061. 
occhi dei Catalano-Aragonesi, i cui giuristi con ogni probabilità erano a conoscenza degli studi di Bartolo da Sassoferrato e di Giovanni da Legnano ${ }^{49}$.

Anche sul secondo punto -il recupero di beni sottratti- vi era corrispondenza tra quanto affermato da Brancaleone Doria e i criteri indicati nel Decretum grazianeo: infatti, il conte di Monteleone in un'altra sua lettera del 3 febbraio 1392 comunicò sempre a Joan de Montbuy la sua intenzione di riprendere tots los lochs et castells que foren per nos dats, los quals malvadament et iniquament ne foren presos [il grassetto è nostro] ${ }^{50}$.

Questa testimonianza di Brancaleone Doria meriterebbe che ci soffermassimo ancora, perché rivela una sorta di contrapposizione giuridica tra le due parti: da un lato i Catalano-Aragonesi che si basavano su due argomenti giuridici: l'infeudazione pontificia del 1297 che aveva attribuito il Regno di Sardegna e Corsica al sovrano aragonese e, il trattato di pace siglato nel 1388, il quale li aveva rimessi in possesso di territori ormai perduti da tempo, non curandosi delle modalità in cui tale documento era stato firmato dalla controparte giudicale. Dall'altro lato, il Doria che si fondava sul concetto di giusta guerra dovuta a soprusi precedentemente subiti.

In considerazione di quanto detto, quindi, si può ipotizzare che il Doria si sentisse legittimato oltre che da un punto di vista morale anche da quello giuridico a intraprendere azioni belliche per il recupero delle fortezze e delle località che erano state restituite al dominio aragonese. Anche se occorre dire che questa presunta legittimazione non sembrò convincere i Sardi giudicali a sostenere in modo acritico la politica del conte di Monteleone.

${ }^{49}$ Per precisione PH. ConTAmine, La guerre au Moyen Âge, p. 450 ricorda che la nozione di principe, del tutto chiara nel mondo imperiale romano, non mantenne la stessa nettezza nel frammentato mondo medioevale, al punto che ciò favorì il formarsi di diverse scuole di pensiero fra i giuristi. A parte l'Ostiense -schierato su posizioni restrittive, che riconoscevano il diritto di dichiarare la guerra solo al papa e all'imperatore- la maggioranza dei giurisperiti estese tale diritto ai principali monarchi europei indipendenti (Castiglia e Francia) e anche a quelli che temporaneamente erano in rapporto di vassallaggio con la Santa Sede come la Sicilia e l'Inghilterra.

${ }^{50}$ ACA, C, Procesos contra los Arborea, vol. IX, f. 57r. Sull'ingiustizia delle clausole del trattato di pace del 1388, Brancaleone Doria aveva fornito altri dettagli nella sua lettera del 29 novembre 1391 citata in precedenza. In essa il conte di Monteleone ricordò al governatore aragonese del Regno di Sardegna che in occasione di un loro precedente incontro a Oristano vos dehim que la pau d'aquesta illa fou feta malvadament et ab gran trassió e violencia da Eximén Peres de Arenòs, ex-governatore, il quale approfittando del fatto che lo teneva prigioniero chiese alla giudicessa Eleonora les terres qui no eren nostres [del Doria], abans eren de la cort d'Arborea. Per quanto riguarda il desiderio di Brancaleone Doria di recuperare con la forza tutte le terre e i villaggi appartenenti al Giudicato di Arborea che erano stati restituiti al re d'Aragona con il trattato del 1388, si può dire che esso avesse un fondamento giuridico nel Digesto, il quale riconosceva che ogni legge e ogni diritto permettevano di opporsi con la forza alla forza esercitata da altri. Cfr. F.H. RuSSELL, The Just War in the Middle Ages, Cambridge, 1975, p. 129 n. 6. 
Diverse sono le testimonianze che si possono addurre in tal senso. Vi è per esempio quella di Nicola Meli, abitante di Sulci (odierna Sant'Antioco) che, a proposito dell'ordine di Brancaleone Doria agli abitanti di Villa di Chiesa (odierna Iglesias), affinché sotto pena corporale e pecuniaria si trasferissero entro le mura della cittadina, riferì che di ciò tota la nació sardescha dona la colpa a micer Brancha et los sab greu ${ }^{51}$.

Testimonianza confermata anche da Antioco Donali, abitante di Palma di Sulci, che, tra le altre cose, affermò di sapere anche lui che il colpevole del nuovo stato di ostilità tra Sardi e Aragonesi era ritenuto micer Brancha, que a tota la nació sardescha sab greu et li.n donen culpa $^{52}$. Affermazioni interessanti, che concorrono con altre a presentare un quadro della società sarda regnicola non del tutto convinta a restare sotto il dominio giudicale a causa della condotta politica del Doria, fermamente deciso a contrastare con le armi il possesso dell'isola agli Iberici. Ce lo conferma in maniera esplicita sempre Antiogo Donali, il quale ricordò ai comissari regi che lo interrogavano che, proprio a causa di questa scontentezza, i sardi regnicoli son stat en punt et son de revellar-se contra ell, et creu que ja ho hagueren fet sino que ell [Brancaleone] los diu que no haian, perque ell se avendrà ab lo senyor rey ${ }^{53}$. Da questa testimonianza sembra dunque emergere che i Sardi avessero deciso di rimanere fedeli al conte di Monteleone scegliendo il male minore, in quanto temevano ancor più la flotta iberica, allestita per debellare definitivamente i baroni siciliani che si opponevano alla signoria catalana. Il timore dei Sardi era stato alimentato ad arte dal Doria, che aveva fatto credere loro che in realtà la flotta fosse diretta in Sardegna per riguadagnare alla Corona quanto la Casa d'Arborea le aveva strappato.

${ }^{51}$ ACA, C, Procesos contra los Arborea, vol. IX, ff. 80r-81v.

${ }^{52}$ ACA, C, Procesos contra los Arborea, vol. IX, f. 81r-v. A queste due testimonianze vogliamo aggiungerne un'altra che riteniamo esemplificativa dell'atmosfera che dovette esservi in Sardegna negli ultimi anni del Trecento. Francesc de Montbuy, governatore del Capo di Logudoro e fratello del governatore generale del Regno di Sardegna, 1'8 novembre 1391 scrisse a fra' Pere Deuna, messaggero della città a Corte, e gli illustrò la situazione che avrebbe poì dovuto riferire. Tra le notizie riportate vi è un accenno alla divisione insorta tra i Sardi in seguito alla notizia dell'allestimento della flotta catalano-aragonese diretta in Sicilia per porre sul trono isolano Martino il Giovane. A ciò aggiunse anche che, poiché Brancaleone Doria aveva imposto alcune nuove tasse, i Sardi si lamentavano di aver perduto la loro precedente libertà e di essersi macchiati del reato di tradimento: Ay mesquins nosaltres; soliam esser franchs con erem del senyor rey et a nos fa tots peytas et traydos Cfr. ACA, C, Procesos contra los Arborea, vol. X, f. $6 \mathrm{v}$.

${ }^{53}$ ACA, C, Procesos contra los Arborea, vol. X, f. 6v.

«Anuario de Estudios Medievales», 33/2 (2003), pp. 849-879 .- ISSN 0066-5061. 
Sempre Brancaleone Doria si rivela un personaggio ricco di spunti per il discorso che stiamo tentando di realizzare. Dai pochi elementi che abbiamo evidenziato finora, sembra che possa essere collocato all'interno di un'atmosfera bellica di tipo cavalleresco anche per quanto da lui scritto in una delle sue numerose missive inviata 1'8 marzo 1393 a Francesc de Montbuy in merito a una battaglia campale che egli di persona avrebbe sostenutonell'eventualità che il re si fosse recato in Sardegna. Il conte di Monteleone disse infatti che il sovrano lo avrebbe trovato en lo camp, ell da una part e nos da l'altra, e aqui patigiarem: se gardarà qui haurà a restituir, restituirà; e qui haurà a tornar, tornarà; e se levores se veurà qui haurà mellor rahó ne mellor justícia $[\ldots]^{54}$. Perfetta descrizione dell'idea che nel Tardo Medioevo si aveva dello scontro frontale tra nemici: in quell'occasione si cristallizzavano tutte le speranze e le paure. A esso, come sottolineava anche il Doria, si affidava il compito di fare giustizia. La vittoria sarebbe andata a chi aveva ragione. E qui, forse, ci si può ricollegare a quanto diceva il conte di Monteleone a proposito della buona e giusta guerra che egli stava conducendo per conto della moglie e in nome del Giudicato di Arborea, privato di terre a esso appartenenti. Anche qui, leggendo in filigrana, si può cogliere qualche aspetto della personalità del conte di Monteleone: dai termini e dal tono usati, possiamo azzardare l'ipotesi che egli si sentisse sicuro di avere la ragione dalla sua parte. Pertanto la vittoria sarebbe stata una logica conseguenza. Andando aldilà della patina cavalleresca e forse anche cortese di dichiarazioni come quelle summenzionate, resta ovviamente da guardare oltre i termini usati per cercare di cogliere quanta propaganda vi fosse in questi scritti del Doria, il quale in più di un'occasione nelle fonti coeve è rappresentato come molto preoccupato dalla possibilità che il sovrano aragonese Giovanni I realizzasse effettivamente la spedizione militare che più volte aveva minacciato. Ma d'altronde, la guerra era uno dei momenti più importanti di teatralizzazione delle società medioevali.

Oltre ai dati che abbiamo riportato in precedenza, prima di concludere vi sono ancora altri elementi relativi alla struttura e all'armamento dell'esercito giudicale su cui intendiamo soffermarci.

Il volume X dei Procesos ci offre qualche spunto in merito. Sempre connessa al tentativo di Brancaleone Doria di impadronirsi della fortezza di Longosardo, di cui abbiamo parlato in precedenza, risulta la testimonianza di

${ }^{54}$ F.C. CASUlA, Carte reali diplomatiche, pp. 79-81.

«Anuario de Estudios Medievales», 33/2 (2003), pp. 849-879 .- ISSN 0066-5061. 
Pietro Staras, abitante di quel villaggio, rilasciata a Valenza il 20 maggio 1393. Nel dettaglio, egli raccontò di essere stato presente nel momento in cui vi fu il tentativo di consegnare la piazzaforte a Brancaleone Doria da parte di Montone e alius sardus vocatus Santolino Mays, conestabulus XXV hominum intus Iudicatum habitans [il grassetto è nostro] ${ }^{55}$. Il tentativò fallì e i due furono interrogati, torturati e condannati a morte. Ma non è questo che ci interessa in questa sede. Piuttosto, l'uso del termine conestabulus, cioè conestabile o comandante di un gruppo di armati che poteva variare da 25 a 50 unità che ci offre un'ulteriore notizie da aggiungere a quelle poche che conosciamo sulla composizione degli eserciti giudicali. Certo, è sempre possibile ammettere che sia stato utilizzato questo termine, in quanto a redigere il verbale degli interrogatori furono scrivani di cultura iberica, e pertanto a conoscenza dell'esistenza della figura del conestabile nell'esercito catalano-aragonese, anche se va detto che il testimone specificò che il Mays era un sardo e perdipiù residente nel territorio giudicale. Possiamo quindi ammettere con una certa verosimiglianza che dovesse comunque sussistere un'unità giudicale di 25 uomini, con a capo un ufficiale definito alla latina conestabile, dal momento che il testimone si prese la briga di indicarlo ai comissari regi che effettuavano gli interrogatori del processo ${ }^{56}$.

Purtroppo per noi le informazioni contenute nelle fonti non sono mai abbondanti, anche se indubbiamente occorrerebbe ripercorrere almeno le principali serie documentarie custodite nell'Archivio della Corona d'Aragona di Barcellona, ove è conservata la stragrande maggioranza dei documenti che interessano la storia sarda dei secoli XIV e XV, per verificare che non sia possibile rinvenire altri dati utili per la ricerca che stiamo conducendo.

$\mathrm{Si}$ rivela molto interessante anche la testimonianza del mercante di Maiorca Pere Barenys, il quale frequentava per motivi di lavoro la costa nordoccidentale della Sardegna compresa tra Alghero e Bosa. Tra le altre cose da lui riportate, vi è la citazione di diversi tipi di armi e della loro riparazione

\footnotetext{
${ }^{55}$ ACA, C, Procesos contra los Arborea, vol. X, ff. 170v-172v.

${ }^{56}$ In questo caso, come anche in molti altri della storia giudicale sarda che non dispone di molte fonti documentarie proprie e che dev'essere ricostruite servendosi delle testimonianze degli "altri", può essere utile seguire le indicazioni fornite da M. FOUCAULT, $L$ 'archéologie du savoir, Paris, 1969 , p. 39 il quale invita a trattare un sistema di pensiero in modo tale da cercare, oltre gli enunciati stessi, le intenzioni del soggetto parlante, la sua attivita cosciente; ciò che egli ha voluto dire, $\mathrm{o}$ ancor meglio, la parte incosciente che si è manifestata suo malgrado in ciò che ha detto. Seguendo le indicazioni dello storico francese, il risultato che si ottiene è di ricostruire un altro discorso, la parola silenziosa che anima dall'interno ciò che noi riusciamo a percepire di primo acchito.
} 
e predisposizione: [...] audivit quod trabuchi parabantur in Castro civitatis bosanis et ipse vidit preparari et muniri castrum et terra manteletis et verdeschis, dixit eciam se vidisse quod continue et sollicite non solum ferrari dicti loci set eciam argenterii clavabant et reparabant coriassias et alia arma $[\ldots]^{57}$.

La descrizione del mercante maiorchino sembra lasciar intendere che tutte le attrezzature da lui menzionate servissero per la difesa della città e del castello di Bosa, secondo porto del Giudicato arborense, quasi che davvero i giudicali temessero eventuali attacchi da parte degli Iberici. Barenys riportò infatti che si stavano preparando alcuni trabucchi -macchine da lancio di pietre o bombe incendiarie, simili alle baliste- mantellets, cioè tavolati laminati di latta che i soldati tenevano dinanzi a sé mentre assaltavano le fortezze per poter avanzare al coperto; e verdesques, costruzioni di legno che normalmente venivano erette dinanzi a torri, mura o ponti per poter coprire i difensori dai colpi dei nemici che assalivan $0^{58}$. Infine, venivano citate anche coriassias e altre armi non meglio precisate.

Un quadro, dunque, perfettamente in linea con quanto avveniva in contemporanea in altri campi di battaglia un po' in tutto il continente europeo.

Altri elementi sugli armamenti degli eserciti giudicali, per quanto non riferiti alla cavalleria, li riscontriamo sempre nei Procesos, e possono servire a dettagliare ulteriormente il quadro che abbiamo tratteggiato finora. A tal fine risulta utile la testimonianza rilasciata il 14 novembre 1391 da Ramon Beneito, abitante di Castel di Cagliari, sulla presa di Villa di Chiesa da parte di Brancaleone Doria. Il testimone riferì, infatti, che il conte di Monteleone salì su un poggio, chiamato Monte Artal, da dove poteva vedere il castello della città pur rimanendo fuori portata delle balestre. Non altrettanto però fece un suo uomo, il quale trovandosi più vicino alla città fu colpito: il risultato fu

${ }^{57}$ ACA, C, Procesos contra los Arborea, vol. V, f. 93 r.

${ }^{58} \mathrm{Un}$ altro riferimento a macchinari da guerra appartenenti ai giudicali è contenuto nella testimonianza di Luigi da Bonifacio, patrono di una barca scoperta, genovese, il quale raccontò di essere stato obbligato dal prefetto di Roma - consuocero del giudice Mariano IV - a portare due trabucchi a Bonifacio o a Terranova. In seguito, dietro richiesta di una persona che si trovava a Civitavecchia per conto del giudice, il prefetto mutò l'ordine, dicendo di portarli a Terranova, dopo aver fatto però scalo a Bonifacio. Così fece, ma nella cittadina corsa fu tenuto un consiglio che decise che le due macchine da guerra non sarebbero state consegnate in Sardegna finché non fosse finita la guerra tra Mariano IV e Brancaleone Doria e dopo la fine dell'assedio di Castelgenovese a opera del giudice. Con la cessazione delle ostilità, il patrono avrebbe dovuto portare le macchine a Bosa; mentre era vicino alla cittadina giudicale, però, fu catturato da Ramon Boter patrono di una nave armata a Castel di Cagliari. Cfr. ACA, C, Procesos contra los Arborea, vol. VIII, ff. $68 \mathrm{v}-69 \mathrm{r}$.

«Anuario de Estudios Medievales», 33/2 (2003), pp. 849-879 .- ISSN 0066-5061. 
que li travassaren lo taulatxo et el braç [il grassetto è nostro] ${ }^{59}$. Simile, ma con qualche dettaglio in più la deposizione di Francesc Oriol, anch'egli abitante della capitale del Regno di Sardegna, il quale raccontò che un giorno ora de vespres, lo dit micer Brancha a peu ab qualque L entaulaxats et ab capellinas et ab la bandera d'Arborea et Doria esteses, acosta.s pres del castell [il grassetto è nostro] ${ }^{60}$.

Ancora, sulla stessa alsariga sebbene riferita a Longosardo, anche la testimonianza di Joan Sexa, soldato di quella villa, il quale tra le altre cose raccontò che lui stesso e i soldati agli ordini di Ponç de Jardí, capitano di quella fortezza, cercarono di colpire con colpi di balestra Brancaleone Doria che era appena smontato da cavallo: et com ell testimoni et los altres del dit laut foren apres del dit micer Brancha desserraren-li dues ballestes, ven VI $o$ VII vegades et aquells qui eren ab lo dit micer Brancha tantost cobriren-lo ab les dargues et taulatxos ${ }^{61}$.

Queste testimonianze ci permettono di acquisire altri dati sull'equipaggiamento dei militari che combattevano sotte le insegne giudicali. Innanzitutto i testimoni descrivono due differenti tipi di scudo usati dalle truppe di terra arborensi. Il taulatxo, o scudo ligneo di forma rotonda, e la darga o adarga, scudo ovale a forma di cuore, realizzato con cuoi disposti in doppio strato cuciti e ribaditi con elementi di metallo, che veniva usato per parare colpi e coprire il corpo, esattamente come riporta la nostra fonte ${ }^{62}$. Ancora, Francesc Oriol aggiunse che i 50 soldati che accompagnavano Brancaleone Doria avevano oltre agli scudi lignei anche le capellinas, sorta di copricapo metallico - ferro o acciaio- indossato dalle truppe di terra armate alla leggera tra XIII e XIV secolo ${ }^{63}$.

Un altro dato ancora, questa volta non sull'equipaggiamento dei soldati, ma sulle unità in cui essi erano suddivisi, lo ricaviamo da una missiva di Brancaleone Doria a Francesc de Montbuy. In questa lettera, datata 8 marzo 1393, il conte di Monteleone scrisse all'ufficiale regio in merito a un

\footnotetext{
${ }^{59} \mathrm{ACA}, C$, Procesos contra los Arborea, vol. IX, ff. $61 \mathrm{v}-65 \mathrm{r}$.

${ }^{60}$ Ibidem, f. $67 \mathrm{v}$.

${ }^{61}$ Ibidem, f. $72 \mathrm{r}-\mathrm{v}$

${ }^{62}$ A.M ${ }^{\text {a }}$. ALCOVER, Diccionari català-valencià-balear, Palma de Mallorca, 1985, tomo I, ad vocem "adarga o darga", p. 180, e tomo X, ad vocem "taulatxo", p. 177.

${ }^{63}$ G.C. Stone, A Glossary of the Construction, Decoration and Use of Arms and Armor in all Countries and in all Times, New York, 1961, ad vocem "capeline", p. 163.

«Anuario de Estudios Medievales», 33/2 (2003), pp. 849-879 .- ISSN 0066-5061.
} 
tale che, dopo essere uscito di prigione, gli prestò omaggio impegnandosi a essere un suo vassallo; in cambio, il conte lo nominò caporal de una bregada que li havem consegnada, la qual bregada està en la nostra cort ${ }^{64}$.

In base a queste informazioni, possiamo forse aggiungere nuovi dati sulla composizione dell'esercito giudicale arborense. Allo stato attuale delle ricerche non siamo in grado di mettere insieme in modo organico i dati che già possedevamo in precedenza - per esempio il sistema delle mudas-con l'esistenza di ulteriori unità come quella delle bregadas appena menzionata, cioè non sappiamo dire se esse fossero collegate -ogni muda, composta da un determinato numero di bregadas, per esempio- o, al contrario, se si trattase di innovazioni alla struttura dell'armata giudicale, apportate forse dallo stesso Brancaleone Doria per far fronte a determinate esigenze belliche. Si può solo sperare che ulteriori rinvenimenti documentari o una rilettura più attenta della terminologia militare nelle fonti già edite possa aiutarci a far luce anche su questo aspetto della società giudicale.

Per concludere, vogliamo solo ribadire che i riflessi di una ricerca sulla cultura e l'organizzazione della guerra nel Giudicato di Arborea fra Trecento e Quattrocento si rivelano estremamente interessanti anche per i singoli dati - per quanto possano essere o sembrare minimi- riguardanti la struttura stessa della società giudicale arborense.

È chiaro che il presente lavoro è ben lungi dall'essere esaustivo. Più che altro, la sua finalità è di sollevare interrogativi che possano alimentare nuovi dibattiti storiografici, che permettano di colmare in qualche modo le notevoli lacune documentarie di cui soffre la storia giudicale. Da questo punto di vista, rimaniamo sempre più convinti che, anche in assenza di fonti inedite, si possano ottenere nuove chiavi di lettura del Medioevo sardo provando ad applicare alle fonti metodologie di lettura e analisi già note che sono proprie delle scienze sociali. Un approccio multidisciplinare, che preveda l'uso di strumenti critici antropologici, sociologici e filosofici permetterebbe -ne siamo sicuri- di acquisire nuovi elementi per tentare di cogliere con più precisione anche ciò che si nasconde al di sotto del primo strato di informazioni contenute nei documenti. Pur rimanendo consapevoli dei rischi insiti in questo tipo di lettura di fonti narrative e documentarie tanto distanti da noi nel tempo, è quanto abbiamo tentato di fare a suo tempo analizzando la figura di Guglielmo II di Narbona, ultimo giudice sardo. Anzi per essere precisi, anche

${ }^{64}$ F.C. CASULA, Carte reali diplomatiche, doc. 51, pp. 79-81.

«Anuario de Estudios Medievales», 33/2 (2003), pp. 849-879 .- ISSN 0066-5061. 
questo presente lavoro è frutto delle riflessioni che iniziammo a fare oltre dieci anni fa, quando ci ponemmo il problema di che tipo di "immagine" della Sardegna e del Giudicato di Arborea dovesse avere il visconte di Narbona e, in contemporanea, di come i sudditi giudicali avessero "visto" il loro nuovo giudice. Da queste semplici domande ne sono derivate tante altre che partendo dalla battaglia di Sanluri hanno finito per riguardare l'intero assetto della società arborense, che ancora nel XIV secolo mostrava tracce del precedente periodo in cui la Sardegna intera era appartenuta all'Impero bizantino. Anche da quest'ultima constatazione -l'isola all'interno dell'impero romano medioevale - scaturiscono molte altre domande che non inseriamo qui perché sono fuori luogo, ma di cui ci stiamo occupando in un'altra sede.

Ritornando agli ultimi secoli del Medioevo, partendo dal tentativo di comprendere se il Giudicato di Arborea fosse dotato o meno di milites nel senso che tradizionalmente si dava a questo termine in quel periodo, i nostri interrogativi ci hanno portato a domandarci che tipo di nobiltà vi fosse all'interno dello stato indigeno sardo e che rapporti eventualmente potesse avere con la militia, nel tentativo di istituire parallelismi con altre realtà esterne all' isola ${ }^{65}$. Altre domande che ci si potrebbe fare sempre sul tema della cavalleria potrebbero riguardare, per esempio, la tipologia delle razze dei cavalli presenti nell'isola durante il Medioevo e la loro struttura fisica, se cioè fossero in grado di sopportare il peso di un cavaliere armato pesantemente. Oppure, come forse è più probabile, se i cavalli sardi fossero più simili a quelli impiegati dagli Arabi nel bacino del Mediterraneo, dalla corporatura non imponente, ben diversi da quelli usati invece nelle regioni dell'Asia Minore che tanta influenza avevano avuto sulla cavalleria pesante bizantina dell'Alto Medioevo.

La presenza di cavalli dalla corporatura robusta, adatti alla guerra, potrebbe portare a interrogarsi -come fa F. Cardini per altre regioni d'Europa - se anche l'attività agraria della Sardegna giudicale dovette conoscere - magari solo in parte- la modifica dei cicli tradizionali di coltura dal biennale romano al triennale diffuso in Gallia ${ }^{66}$.

Altri dati potrebbero essere ottenuti dal proseguimento dell'esplorazione di un altro filone di ricerche pertinenti al mondo della letteratura cortese. Sono già stati condotti alcuni studi sui riferimenti al mondo

${ }^{65}$ L. GALliNARI, Gli ultimi anni di esistenza, attualmente in corso di stampa.

${ }^{66} \mathrm{~F}$. CARDINI, Alle radici della cavalleria, p. 267.

«Anuario de Estudios Medievales», 33/2 (2003), pp. 849-879 .- ISSN 0066-5061. 
giudicale sardo presenti in diversi componimenti di poeti provenzali, siciliani e toscani -alcuni dei quali li abbiamo ricordati anche in precedenza - che consentono allo studioso di cogliere la molteplicità di legami che unirono le società giudicali isolane sia al resto del continente italiano, sia ad altre aree dell'Europa mediterranea che in quei secoli erano all'avanguardia nell'attività poetica $^{67}$. Questi componimenti, pur valutandoli nella dovuta ottica, con il metodo di lettura critica proposto da Foucault possono offrire nuovi e fecondi spunti per cogliere le immagini culturali che i singoli artisti coglievano della realtà sarda o, forse, quelle che dall'interno dell'isola volutamente o meno si proiettavano.

${ }^{67} \mathrm{Sul}$ rapporto tra la Sardegna giudicale e la poesia trovatorica rimandiamo oltre che ai già citati lavori di Paolo Maninchedda, anche a A.M. OLIVA, Guglielmo di Massa «Al prou marques de Sardenha qu'ab joi viu et ab sen renha», in Gli Obertenghi di Massa e della Lunigiana ed $i$ regni della Sardegna (secoli XII-XIV), Massa, 1999, pp. 85-108.

*Anuario de Estudios Medievales*, 33/2 (2003), pp. 849-879 .- ISSN 0066-5061. 